\title{
Performance Trade-offs of an Interferometric Micro-g Resolution Accelerometer
}

\author{
Maximillian Perez \\ Mechanical and Aerospace Eng. \\ University of California, Irvine \\ Irvine, California 92697-3975 \\ Email: max.perez@uci.edu
}

\author{
Andrei M. Shkel \\ Mechanical and Aerospace Eng. \\ University of California, Irvine \\ Irvine, California 92697-3975 \\ Email: ashkel@uci.edu
}

\begin{abstract}
A high resolution bulk micromachined accelerometer based on transmission-type intrinsic Fabry-Perot interferometer has been experimentally evaluated via direct inertial characterization for the first time. The device characterization includes frequency and time domain evaluation. The sensor characteristics of bandwidth, range, sensitivity, and resolution are obtained and the trade-offs between these performance characteristics are examined. The sensor performance, which approaches its theoretical limit with a maximum resolution of $2.9 \mu \mathrm{g}$ is obtained, demonstrating inertial navigation grade performance.
\end{abstract}

\section{INTRODUCTION}

Multiplexed sensor systems are of broad interest, ranging from pervasive infrastructure monitoring to complex system control. This group has proposed [1] and demonstrated [2] the passive optical multiplexing of transmissive interferometric sensors by engineering the optical properties of each sensor to allow linear serialization along an optical path. This paper focuses on the detailed analysis of a single sensor of such a series and evaluates the performance trade-offs. This work provides tools required to characterize and demonstrate the capabilities of transmission-type intrinsic Fabry-Perot accelerometers.

\section{BACKGROUND}

The characteristics of passive, seismic proof-mass, micromachined accelerometers based on Fabry-Perót interferometers (FPI) are developed and characterized. The devices are formed from pairs of etched substrates with mirrored surfaces, which are micro-assembled by epoxy bonding to form a gap of parallel faces (Figs. 1 and 2). The proof-mass mirror substrate is composed of a 'thinned-wafer' flexure connecting the proofmass to a frame. The reference mirror substrate contains a similar structure where the flexure has been replaced by fixed supports to minimize compliance (Fig. 3). High performance devices require structures with high gap aspect ratios, smooth surfaces and no perforations. In such structures squeeze film characteristics are observed and are included in the dynamic models. In the structures studied, the proof-mass radius $r$ ranges from 0.5 to $1.5 \mathrm{~mm}$ with an optical gap $d_{\text {gap }}$ ranging from 15 to $120 \mu \mathrm{m}$. The fabrication of such devices have been introduced elsewhere [3], [4].

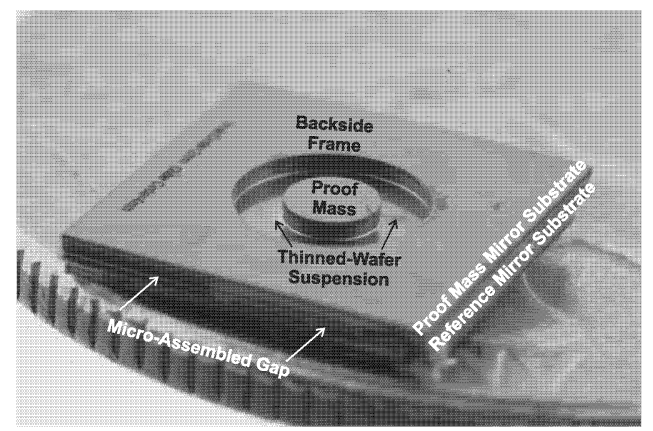

Fig. 1. Micro-fabricated optical FPI-based accelerometer (on a US quarter)

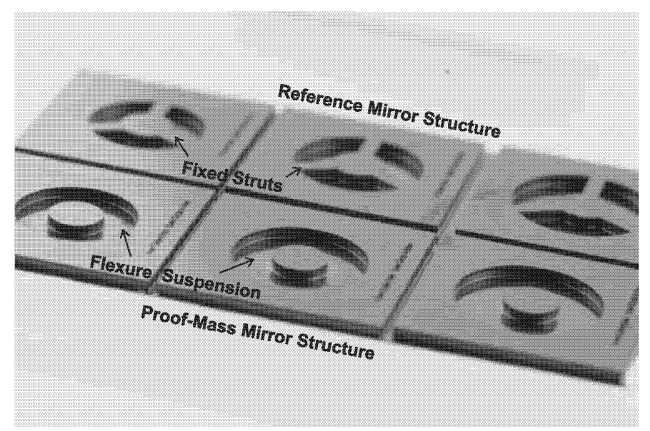

Fig. 2. An array of FPI accelerometer component pairs

\section{A. Mechanical Response}

Under external acceleration, the mechanical response of the sensor is modeled as a lumped mass-spring-damper system and is written in the Laplace domain by

$$
\frac{Z(s)}{s^{2} Y(s)}=\frac{Z(s)}{A_{y}(s)}=\frac{-m}{m s^{2}+c_{s f} s+k_{t o t}},
$$

where $m$ is is the mass of the sensing element, $c_{s f}$ is the effective damping, $k_{t o t}$ is the total stiffness composed of the components due to the flexure suspension $k$ and the squeeze film $k_{s f}$, where $k_{t o t}=k_{s f}+k$. $Y$ is the base displacement, $X$ is the proof mass displacement, $Z$ is relative displacement between the two, and $A_{y}$ is the measured base acceleration (Fig. 4). When normalized by mass, the parametric model is 


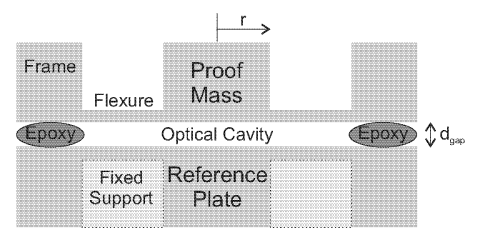

Fig. 3. Cross-section of the assembled device

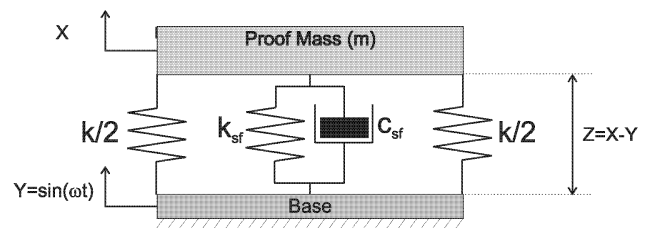

Fig. 4. Lumped parameter model of the passive accelerometer with flexure suspension stiffness $(k)$, squeeze film stiffness $\left(k_{s f}\right)$ and damping $\left(c_{s f}\right)$

expressed as

$$
\frac{Z(s)}{A_{y}(s)}=\frac{-1}{s^{2}+2 \omega_{n} \zeta s+\omega_{n}^{2}},
$$

where the angular natural frequency $\omega_{n}=\sqrt{k_{t o t} / m}$ and the damping ratio $\zeta=c_{s f} /\left(2 m \omega_{n}\right)$. Such sensors are designed to operate at frequencies in the flat response region of the frequency domain below their natural frequency, with the lowfrequency response derived from (2) when $s \rightarrow 0$ as

$$
z=-(m / k) a_{y}=-\frac{1}{\omega_{n}^{2}} a_{y}
$$

\section{B. Optical Response}

The operation of the FPI-based inertial sensor has been explained in depth previously [5]. Briefly, the spectral position of an interferometric fringe created by an optical cavity between two reflective surfaces provides a high-precision pickoff (sensing) mechanism for the spacing between the surfaces (Fig. 5). If one surface is the face of a proof mass and the other is an inertial reference, the deflection between the plates

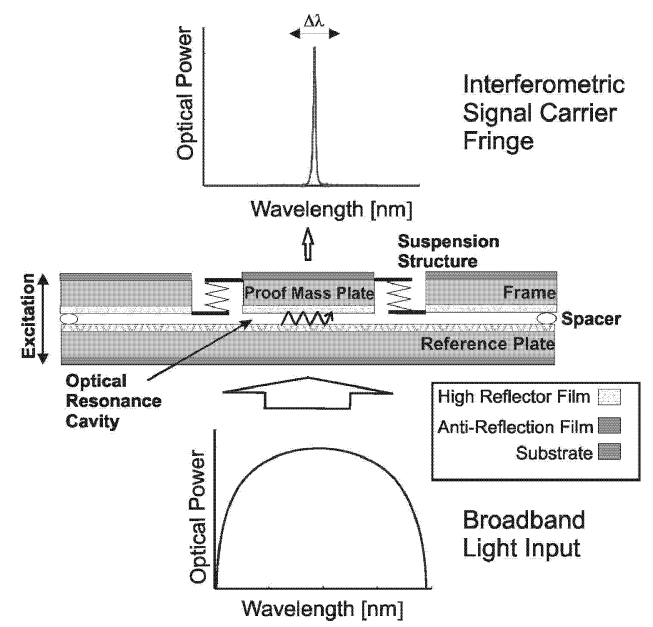

Fig. 5. Optical operation of the Fabry-Perót based inertial sensor

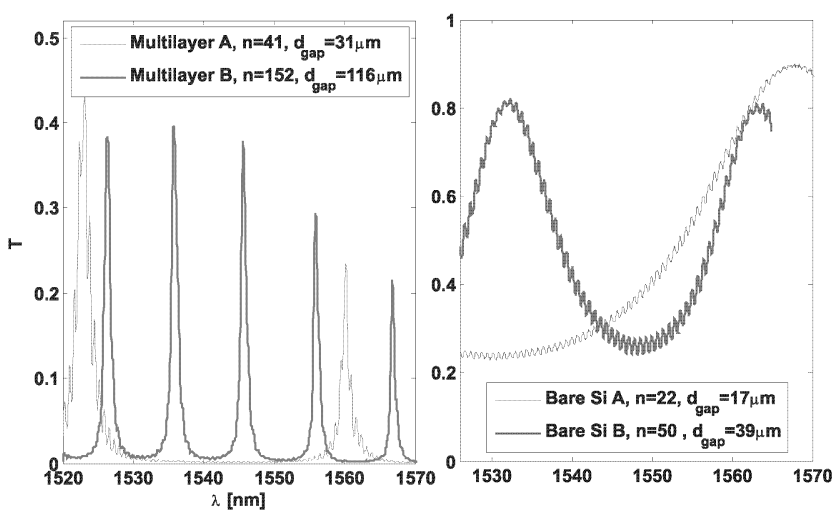

Fig. 6. Optical response (Transmittance) of devices with (left) multilayer reflectors and (right) bare silicon cavity reflectors

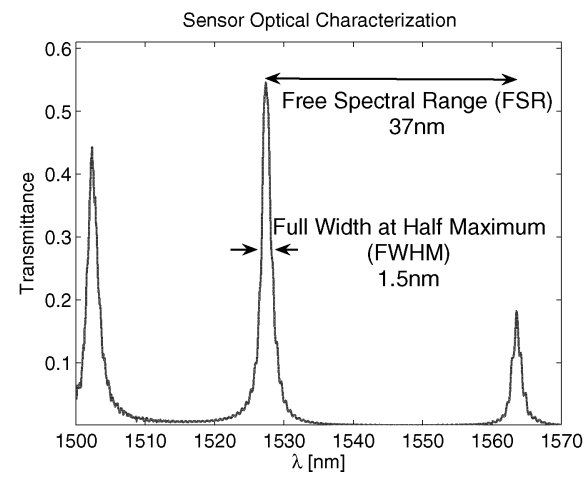

Fig. 7. Experimental optical characteristics of the characterized sensor indicating an optical finesse $\left(N=\frac{F S R}{F W H M}\right)$ of 25 on the $n=41^{\text {st }}$ order fringe and a plate gap spacing of $d_{\text {gap }}=31.3 \mu \mathrm{m}$

is related to the base acceleration by the fringe position shift according to (4):

$$
a=\left[\frac{A_{y}(s)}{Z(s)}\right] \frac{n\left(\lambda-\lambda_{i}\right)}{2},
$$

where $n$ is the optical order of the FPI, $\lambda_{i}$ is the initial wavelength position of the fringe when the sensor is not excited, and $\lambda$ is the wavelength of the displaced fringe under acceleration $a$. The acceleration is monitored by the modulation of the transmitted optical power, with a laser, by

$$
a=-\omega_{n}^{2} \frac{n}{2}\left[\frac{\delta \lambda}{\delta T}\right]_{\lambda_{0}} \frac{1}{P_{\lambda_{0}}} \Delta P,
$$

where $[\delta T / \delta \lambda]_{\lambda_{0}}$ is the slope of the fringe, $P_{\lambda_{0}}$ is input optical power at the wavelength $\lambda_{0}$ and $\Delta P$ is the power modulation.

\section{CHARACTERIZATION}

\section{A. Optical}

The intrinsic reflectance of a polished silicon wafer is sufficient to create an optical cavity capable of forming FPIbased inertial sensors. However, there are trade-offs in the performance of such a device. The slope of an optical fringe is proportional to the resolving power of an FPI, such that

$$
[\delta T / \delta \lambda]_{\max } \propto N \times n,
$$




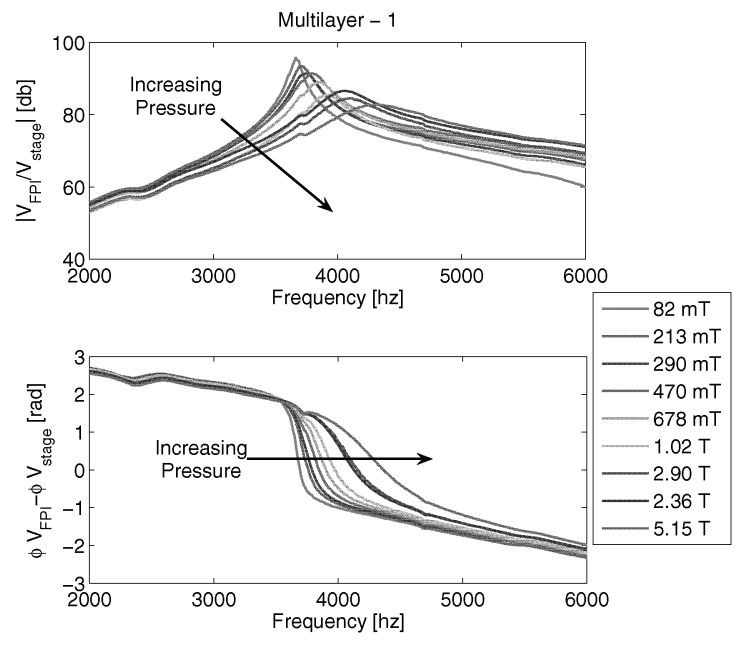

Fig. 8. Frequency response of the characterized sample under basedisplacement excitation under vacuum

where $N$ is the optical finesse of the FPI. The finesse is known to be a strong function of the reflectance of the cavity surfaces ( $N=f\left(R_{s}\right)$ ) and the fringe order is dependent on the gap spacing ( $n=\left(2 d_{\text {gap }}\right) / \lambda_{o}$ in air) [6]. However, using (6) with (5), the functional dependence of the FPI-based accelerometer on fringe order $n$ is eliminated and

$$
a \propto-\frac{\omega_{n}^{2}}{2 N} \frac{1}{P_{\lambda_{0}}} \Delta P,
$$

indicating that only increasing the cavity surface reflectance (not gap spacing) can increase instrument optical sensitivity.

In order to form higher resolution devices, optical coatings must be deposited onto the optical surfaces. In this work, thin film multilayers of PECVD of amorphous $\mathrm{Si}$ and $\mathrm{SiO}_{2}$ are used to both increase the cavity reflectance and to provide the capability for device serialization [2]. Figs. 6 and 7 show the optical characteristics of such devices.

\section{B. Mechanical}

In order to resolve the mechanical characteristics of samples, a unique vacuum vibration chamber was developed with a view-through optical path. In general, the damping force and spring force of a squeeze film will increase with pressure, resulting in a characteristic shift to a higher resonant frequency and peak broadening, as in Fig. 8. Using this experimental system, the squeeze film effects due to squeeze film damping $\left(c_{s f}\right)$ and stiffness $\left(k_{s f}\right)$ were reduced allowing the resonate frequencies to be resolved. Using a swept frequency analysis under vacuum with variable base excitation and controlled sample response (constant proof-mass displacement), the frequency response of the sample was obtained. The $\frac{\pi}{2}$ phase cross-over and peak amplitude indicate the fundamental natural frequency $f_{n}=\omega_{n} / 2 \pi$.

\section{PERFORMANCE}

The characteristics of an FPI-based accelerometer are described below.

\section{A. Sensor Bandwidth}

FPI-based sensor bandwidth can be defined by the frequency range in which equation (3) is accurate to within an established error. This is the region of 'flat' low-frequency response that is below the main resonance. Bandwidth is most strongly dependent on the structural natural frequency. For the most commonly encountered case of nonzero, sub-critical damping $(0<\zeta<1)$, the bandwidth can be estimated as a simple fraction of the natural frequency. For better than $1 d B$ of response linearity, the bandwidth is estimated as

$$
\Delta f_{B W} \simeq \frac{1}{3} \frac{\omega_{n}}{2 \pi}
$$

From Fig. 8, $f n=\omega_{n} / 2 \pi \geq 4.4 k H z$ yielding a bandwidth estimate for ambient pressure of $\Delta f_{B W} \geq 1.45 \mathrm{kHz}$ [7].

\section{B. Sensor Dynamic Range}

One feature of FPI-based sensors is that multiple families of fringes are present in the spectral response and are available for carrying pick-off signals. However, all fringes carry the same information. Thus, the range of the device is limited to half the symmetric optical range between the fringes or free spectral range (FSR). From (4), the maximum dynamic range can be calculated as

$$
\Delta a_{\max }=\omega_{n}^{2} \frac{n}{4} F S R
$$

However, the response is linearized about an operating wavelength on a fringe resulting in a linear dynamic range $\Delta a_{\text {lin }}<$ $\Delta a_{\max }$. Thus, $\Delta a_{\text {lin }}<\Delta a_{\max }=0.75 \mathrm{~g}$.

\section{Sensor Sensitivity}

The sensitivity or scale-factor of a sensor is the linearized factor relating the measurand to signal. From (5), the scalefactor is

$$
\left|\frac{\Delta P}{\Delta a}\right|=\frac{2}{n \omega_{n}^{2}}\left[\frac{\delta T}{\delta \lambda}\right]_{\lambda_{0}} P_{\lambda_{0}}
$$

when evaluated at the fringe half maximum $\left[\frac{\delta T}{\delta \lambda}\right]_{-3 d B}=$ $0.7 \mathrm{~nm}^{-1}$ for a $P_{\lambda 0}=1 \mathrm{~mW}$ laser, $\left|\frac{\Delta P}{\Delta a}\right|=4.6 \mu \mathrm{W} / \mathrm{g}$.

\section{Sensor Resolution}

Signal noise of a passive inertial sensor estimated from the noise due to thermal-mechanical motion of the proof mass. The acceleration resolution limit is

$$
a_{l i m}=\sqrt{\frac{8 k_{B} T \zeta \omega_{n}}{m}},
$$

where $k_{B}$ is Boltzmann's constant and $T$ is the absolute temperature measured in Kelvin [3]. Although multiple other possible noise sources exist in the electrical and optical instrumentation that may limit the resolution of the sensor, the thermal-mechanical noise can be expected to provide the lower bound of resolution limit.

The quantities needed to evaluated (11) are extracted from the resonate frequency response of the sample under vacuum. Under atmospheric conditions where a resonate peak could not be resolved, both $k_{s f}$ and $c_{s f}$ are expected to increase. This 


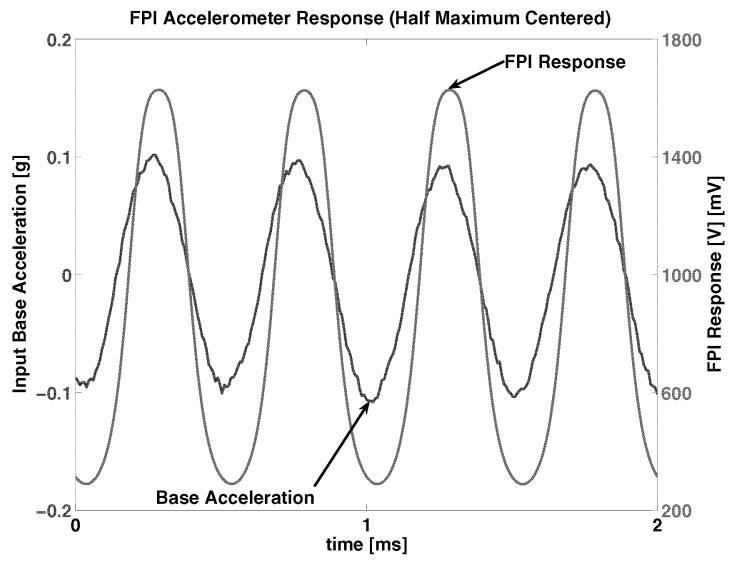

Fig. 9. Base-excited time domain characterization of the sample at $2 \mathrm{kHz}$

will increase both $\zeta$ and $\omega_{n}$ and, correspondingly, increase $a_{m i n}$. Thus, the dynamic characterization from Fig. 8 can be used to estimate a lower bound of the acceleration resolution. For $\zeta=0.075$ and $\omega_{n}=27.6 \mathrm{krad} / \mathrm{s}$ (estimated from the response under five Torr), yields $a_{\min }=0.7 \mu \mathrm{g}$.

\section{EXPERIMENTAL EVALUATION}

The sample is evaluated under sinusoidal base excitation at $2 \mathrm{kHz}$ under atmospheric pressure. A $1 m W$ tunable laser (HP $8168 \mathrm{E}$ ) is passed through the sample. The transmitted power is monitored as a voltage via photodetector with an integrated transresistance amplifier (ThorLabs PDA255). A lowresolution commercial accelerometer on the same platform is used to estimate a reference signal (Fig. 9). Eliminating time between the signals, two linear regions on the response curve are identified (Fig. 10).

Selection of the detection region and operating wavelength defines the trade-offs between sensitivity and the range of operation. To maximize sensitivity, the wavelength at the half maximum of the fringe is monitored yielding a sensitivity of $8.7 \mathrm{~V} / \mathrm{g}$ that is linear within $20 \%$ for a full scale of $0.1 \mathrm{~g}$. To maximize range, the wavelength at $1 / 4$ FSR between fringes is monitored yielding a sensitivity of $43 \mathrm{mV} / \mathrm{g}$ within $20 \%$ for $0.8 \mathrm{~g}$. The noise floors of the zero input signals in both operating modes were observed to be 0.32 and $0.56 \mu \mathrm{V} / \sqrt{\mathrm{Hz}}$, respectively. This yields a maximum resolution of $2.9 \mu \mathrm{g}$ for narrow range and of $330 \mu \mathrm{g}$ for wide range operation at $2 \mathrm{kHz}$. This suggests that the sensor may be suitable for narrow-range super high performance (i.e., inertial navigation) and widerrange high performance (i.e., guidance) applications [8].

The maximum experimental resolution observed is within an order of magnitude of the theoretical limit. The expected optical signal noise from the photodetector at the excitation frequency is $1.3 p \mathrm{~W}$. This yields a voltage noise of $12 \mu \mathrm{V}$, which is close to the $14 \mu \mathrm{V}$ noise experimentally observed at $2 k H z$. Thus, the resolution deviation from that theoretically predicted is largely due to the noise in the photodetector.

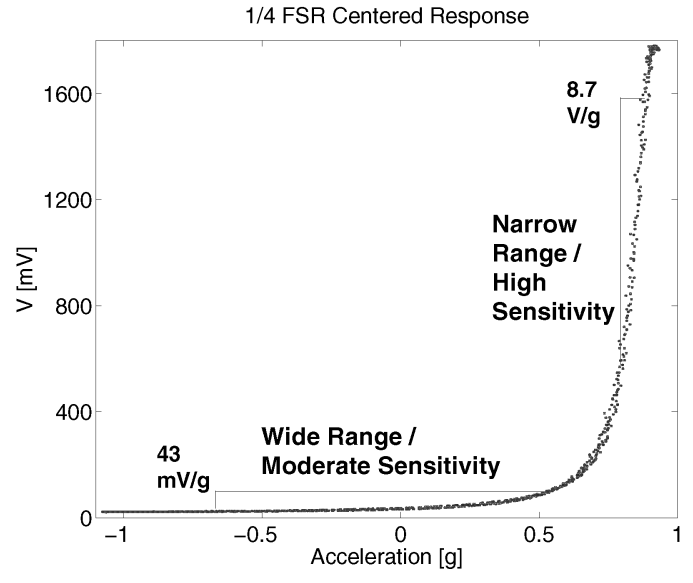

Fig. 10. Acceleration sense characterization of the sample at $2 \mathrm{kHz}$

\section{CONCLUSION}

The operation and dynamic evaluation of the transmissiontype FPI-based accelerometer has been presented. Both the optical and mechanical characteristics were independently evaluated and used to predict sensor performance. The dependence of the performance characteristics from natural frequency show the trade-off between improving bandwidth and dynamic range at the cost of sensitivity and resolution. The operational demonstration of a sensor showed the tradeoffs between range and sensitivity with respect to the optical properties and the choice of the pick-off wavelength. The device was experimentally determined to have a maximum resolution approaching the theoretical limit allowing for use as a high grade inertial sensor over a frequency bandwidth greater than $1 \mathrm{kHz}$. Accordingly, the optical sensor has sensing properties such that it is suitable for use in the previously mentioned sensor networks.

\section{ACKNOWLEDGMENT}

This work is supported in part by NSF, grant CMS-0223050.

\section{REFERENCES}

[1] M. A. Perez and A. M. Shkel, "Conceptual design and preliminary characterization of serial array system of high-resolution MEMS accelerometers with embedded optical detection," Smart Structures and Systems, vol. 1, no. 1, December 2004.

[2] M. Perez and A. M. Shkel, "Passive network of FabryPerot based sensors with wavelength multiplexing capabilities," SPIE Smart Structures and Materials Conference Proceedings, February 2006.

[3] E. J. Eklund and A. M. Shkel, "Factors affecting the performance of micromachined sensors based on fabryperot interferometry," Journal of Micromechanics and Microengineering, vol. 15, pp. 1770-1776, July 2005.

[4] M. A. Perez, E. J. Eklund, and A. M. Shkel, "Designing micromachined accelerometers with interferometric detection," IEEE Sensors 2005 Conference Proceedings, September 2005.

[5] E. Udd, "Fiber optic smart structures," Proceedings of the IEEE, vol. 84, no. 6, pp. 884-894, June 1996.

[6] P. D. Atherton, N. K. Reay, J. Ring, and T. R. Hicks, "Tunable FabryPerot filters," Optical Engineering, vol. 20, no. 6, pp. 806-814, 1981.

[7] H. Eren, Acceleration, Vibration, and Shock Measurement. CRC Press LLC, 2000, ch. 17.

[8] IEEE recommended practice for precision centrifuge testing of linear accelerometers, Std 836-2001 ed., IEEE, 2001. 